\title{
What is the Best Way to Treat Left Main Coronary Artery Disease?
}

\author{
Takaaki Katsuki, MD
}

$\mathbf{N}$

umerous researchers have attempted to improve the methods of percutaneous coronary intervention (PCI), with the goal of obtaining equivalent or better long-term results compared with coronary artery bypass graft (CABG). In the beginning of the drug eluting stent (DES) era, the initial success rate was very high rate and the target lesion revascularization (TLR) of PCI was dramatically improved. However, unprotected left main coronary artery (ULMCA) lesions, chronic total occlusion, bifurcated lesions and diffuse coronary atherosclerosis are still major problems in the PCI field.

\section{Article p 705}

At the 2008 European Society of Cardiology (ECS) in Munich, the results of the SYNTAX (SYNergy between PCI with TAXUS and cardiac surgery) trial were reported. The SYNTAX randomized trial was a comparison of the utility of contemporary CABG and PCI with drug-eluting TAXUS ${ }^{\text {TM }}$ Express $^{2 \mathrm{TM}}$ Stents in patients with left main and/or 3 vessel coronary disease. In the randomized SYNTAX cohort, there were comparable overall safety outcomes (death, cerebrovascular accident (CVA), myocardial infarction (MI)) in CABG and PCI patients at 12 months (7.7 vs $7.6 \%$ ). There was a significantly higher rate of revascularization in the PCI group (13.7 vs 5.9\%), and a significantly higher rate of CVA in the CABG group (2.2 vs $0.6 \%$ ). The incidence of overall major adverse cardiac cerebellar events in the PCI group was higher (17.8 vs $12.1 \%$ ) due to an excess of redo revascularization compared with $\mathrm{CABG}$.

There have been several studies comparing the short- or medium-term follow-up data of ULMCA lesions treated with PCI or CABG in a non-randomized form. Two of these studies, that is, those of Lee et $\mathrm{al}^{1}$ and Chieffo et al, reported results roughly identical to those of the SYNTAX randomized trial. Bravata et al also reviewed the comparative effectiveness of PCI and CABG by meta-analysis? This review concluded that $\mathrm{CABG}$ was more effective in relieving angina and led to fewer repeated revascularizations but had a higher risk for procedural stroke. Survival to 10 years was similar for both procedures.

Several studies from Taiwan, Korea and Japan have reported excellent long-term results obtained by PCI. We

The opinions expressed in this article are not necessarily those of the editors or of the Japanese Circulation Society.

(Received December 24, 2008; accepted December 25, 2008)

Division of Cardiovascular Medicine, Jichi Medical University, Shimotsuke, Japan

Mailing address: Takaaki Katsuki, MD, Division of Cardiovascular Medicine, Jichi Medical University Hospital, 3311-1 Yakushiji, Shimotsuke 329-0498, Japan. E-mail: katsukit@jichi.ac.jp

All rights are reserved to the Japanese Circulation Society. For permissions, please e-mail: cj@j-circ.or.jp previously reported the feasibility and safety of transradial stenting for ULMCA stenosis. The 1-year TLR rate of left main coronary lesions and cardiac death rate were $14.2 \%$ and $3.5 \%$, respectively. Park and Kim reported acceptable long-term outcomes of PCI with DES to treat ULMCA stenoses. Seung et al compared the long-term treatment effects of coronary stenting and CABG in a large-scale registry? They enrolled 1,102 patients treated with stents and 1,138 patients treated with $\mathrm{CABG}$. There was no significant difference between the stenting and CABG groups in the risk of death or the risk of a composite outcome. In non-bifurcation lesions of the ULMCA, PCI with drug-eluting stents appeared safe and achieved extremely good long-term results, with a long-term major adverse clinical event rate of $7.4 \%$ and a restenosis rate of $0.9 \%$ ? There was thus a tendency toward low TLR rates in the Asian reports. These impressive results were likely attributable to the strategies and techniques used in the stent inplant, that is, high pressure post-dilation, shorter stent length, lower stent numbers, frequent use of IVUS and kissing balloon technique. In the future, a large-scale follow-up registry will be needed for far-east countries.

The results of this manuscript ${ }^{8}$ were consistent with those of the SYNTAX trial and the reports from Korea. High rates of stroke, death and in-hospital MACE were observed after the procedure in the $\mathrm{CABG}$ group, and high rates of postprocedural MI were observed in the PCI group. The CABG group showed excellent results in TLR-free and revascularization-free survival compared with the PCI group. But the DES group had the highest overall survival rate. PCI is an effective alternative for the management of patients with ULMCA stenosis. The next goal of PCI is to minimize the TLR of ULMCA lesions.

\section{References}

1. Lee MS, Kapoor N, Jamal F, Czer L, Aragon J, Forrester J, et al. Comparison of coronary artery bypass surgery with percutaneous coronary intervention with drug-eluting stents for unprotected left main coronary artery disease. J Am Coll Cardiol 2006; 47: 864-870.

2. Chieffo A, Morici N, Maisano F, Bonizzoni E, Cosgrave J, Montorfano $\mathrm{M}$, et al. Percutaneous treatment with drug-eluting stent implantation versus bypass surgery for unprotected left main stenosis: A singlecenter experience. Circulation 2006; 113: 2542-2547.

3. Bravata DM, Gienger AL, McDonald KM, Sundaram V, Perez MV, Varghese R, et al. Systematic review: The comparative effectiveness of percutaneous coronary interventions and coronary artery bypass graft surgery. Ann Intern Med 2007; 147: 703-716.

4. Cheng CI, Wu CJ, Fang CY, Youssef AA, Chen CJ, Chen SM, et al. Feasibility and safety of transradial stenting for unprotected left main coronary artery stenosis. Circ J 2007; 71: 855-861.

5. Park SJ, Kim YH. Percutaneous coronary intervention as an alternative to bypass surgery for unprotected LMCA stenosis. Expert Rev Cardiovasc Ther 2008; 6: 1107-1114.

6. Seung KB, Park DW, Kim YH, Lee SW, Lee CW, Hong MK, et al. Stents versus coronary-artery bypass grafting for left main coronary artery disease. $N$ Engl J Med 2008; 358: 1781-1792. 
7. Chieffo A, Park SJ, Valgimigli M, Kim YH, Daemen J, Sheiban I, et al. Favorable long-term outcome after drug-eluting stent implantation in nonbifurcation lesions that involve unprotected left main coronary artery: A multicenter registry. Circulation 2007; 116: 158-162.
8. Cheng CI, Lee FY, Chang JP, Hsueh SK, Hsieh YK, Fang CY, et al. Long-term outcomes of intervention for unprotected left main coronary artery stenosis: Coronary stenting vs coronary artery bypass grafting. Circ J 2009; 73: 705-712. 